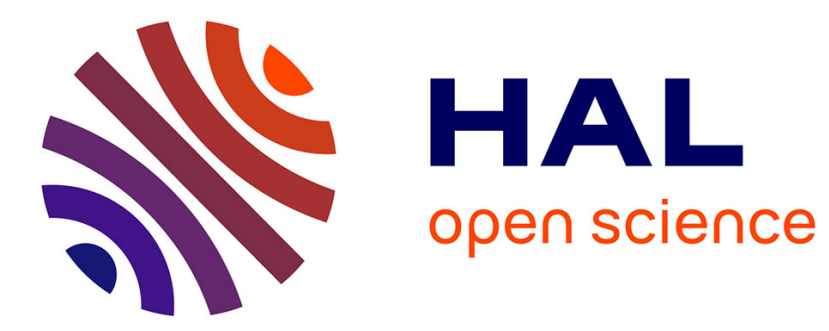

\title{
La rupture du contrôle par la confiance dans les relations client-fournisseur
}

Gwenaëlle Nogatchewsky, Rachel Beaujolin-Bellet

\section{To cite this version:}

Gwenaëlle Nogatchewsky, Rachel Beaujolin-Bellet. La rupture du contrôle par la confiance dans les relations client-fournisseur. Comptabilité - Contrôle - Audit, 2005, 11 (2), pp.39-60. halshs-00143134

\section{HAL Id: halshs-00143134 \\ https://shs.hal.science/halshs-00143134}

Submitted on 8 Jul 2010

HAL is a multi-disciplinary open access archive for the deposit and dissemination of scientific research documents, whether they are published or not. The documents may come from teaching and research institutions in France or abroad, or from public or private research centers.
L'archive ouverte pluridisciplinaire HAL, est destinée au dépôt et à la diffusion de documents scientifiques de niveau recherche, publiés ou non, émanant des établissements d'enseignement et de recherche français ou étrangers, des laboratoires publics ou privés. 


\title{
LA RUPTURE DU CONTROLE PAR LA CONFIANCE DANS LES RELATIONS CLIENT- FOURNISSEUR
}

\author{
Rachel Beaujolin-Bellet \\ Gwenaëlle Nogatchewsky
}

\begin{abstract}
Résumé
Le contrôle par la confiance dans les relations client-fournisseur est à la fois puissant ... et fragile. A partir d'une étude de cas concernant des relations client-fournisseur dans la vallée de la Maurienne, nous étudions un processus de rupture du contrôle par la confiance, ses causes et ses conséquences pour les partenaires. Il en ressort que le contrôle par la confiance, lorsqu'il est affranchi des dispositifs formels, fragilise les relations car il n'apporte pas la preuve de leur performance.
\end{abstract}

Mots clés : contrôle inter-organisationnel - relation client-fournisseur - confiance

\begin{abstract}
Breach of trust control in buyer-supplier relationships

Trust control in buyer-supplier relationships is both powerful ... and fragile. From a case study concerning buyer-supplier relationships in the Maurienne valley (France), we study a process of breach of trust control, its causes and its consequences for the partners. It brings out that trust control which does not rely on formal devices weakens the relationships because it cannot prove their performance.
\end{abstract}

Keywords : inter-organisational control, buyersupplier relationship, trust

\section{Correspondance :}

Rachel Beaujolin-Bellet, Professeur, Reims-Management School, 59 rue Pierre Taittinger BP 30251061 Reims Cedex, 03267747 14, rachel.beaujolin@reims-ms.fr.

Gwenaëlle Nogatchewsky, Maître de conférences, DRM-Crefige, CNRS UMR 7088, Université ParisDauphine, Place du Maréchal de Lattre de Tassigny 75775 Paris Cedex 16, 0140860622.

nogatchewsky@yahoo.fr 


\section{Introduction}

L'externalisation croissante des activités des entreprises pose le problème du management des relations client-fournisseur, et plus particulièrement du contrôle nécessaire à leur bon fonctionnement. Concernant la problématique du contrôle inter-organisationnel, la théorie des coûts de transaction (TCT), qui propose une dichotomie entre le marché et la hiérarchie, occupe sur le plan théorique une position dominante (Froehlicher, 2000). Toutefois, le développement de formes de coopération stables telles que le partenariat ou le réseau appelle de nouvelles explications théoriques (Dekker, 2004). En effet, ces systèmes d'échange coopératifs reposent sur la confiance, la réciprocité, la collusion tacite, l'absence relative de comportement calculateur et opportuniste et la TCT ne permet pas d'expliquer leur mode de gouvernance (Larson, 1992). Dès lors, à partir des travaux sur la coopération de Richardson (1972), sur ceux concernant l'échange relationnel de Macneil (1980), sur le clan d'Ouchi (1980) ou sur l'encastrement social de Granovetter (1985), un certain nombre de chercheurs ont proposé une autre voie pour décrire et expliquer ces situations de contrôle qui ne reposent pas sur des mécanismes visibles de la coopération. Il s'agit du contrôle par la confiance certains parlent plutôt de contrôle social ou de clan.

La confiance a fait l'objet d'un très grand nombre de travaux de recherche dans la littérature inter-organisationnelle. Les chercheurs s'attachent à en expliquer les fondements, le processus de développement et les implications (Guibert, 1999) ${ }^{1}$. Ainsi, il est aujourd'hui largement reconnu que la confiance émerge des interactions sociales entre les partenaires d'échange et se construit dans la durée. Elle est source d'avantage compétitif parce qu'elle permet 1) de limiter les risques liés aux échanges en situation d'incertitude, 2) de dissuader les partenaires de se comporter de manière opportuniste, 3) de réduire les coûts de transaction et 4) d'améliorer la qualité de la coopération (efforts joints, flexibilité, adaptation, partage d'information). Toutefois, la confiance présente également des risques pour les partenaires qui sont principalement liés à la vulnérabilité de l'un face à l'opportunisme éventuel de l'autre. Bien que reconnaissant ces risques, peu de chercheurs s'interrogent sur les fragilités des fondements de la confiance et ils sont encore moins nombreux à conduire des recherches empiriques sur les processus de destruction de la confiance entre partenaires. A notre connaissance, seuls Dwyer et al. (1987) intègrent dans leur analyse théorique une phase de 
dissolution de la coopération, mais ils n'envisagent pas concrètement les conséquences d'une telle rupture pour les parties.

L'objet de cet article est ainsi d'apporter des éléments théoriques et empiriques de réponse aux questions : Comment comprendre la rupture du mode de contrôle par la confiance dans des relations client-fournisseur? Quelles sont les fragilités inhérentes à ce mode de contrôle ? Et quelles peuvent être les implications de cette rupture pour les clients et pour les fournisseurs?

Nous considérons, à l'instar de Chiapello (1996), qu'un mode de contrôle est un ensemble de facteurs d'influence qui peuvent être visibles ou invisibles, intentionnels ou non. Ainsi, dans les relations client-fournisseur, les modes de contrôle recouvrent à la fois les dispositifs mis en place par les partenaires pour maîtriser la coopération et les facteurs d'influence plus informels qui émergent des interactions entre les acteurs et de leur inscription au sein de réseaux sociaux.

Cette recherche s'appuie sur une étude de cas réalisée dans le bassin de la Maurienne. Ce cas a été choisi parce que, au milieu des années 1990, des clients ont rompu les relations de confiance qu'ils entretenaient avec leurs fournisseurs de sous-traitance d'entretien et de maintenance pour privilégier des relations basées sur des critères de performance standardisés répondant aux normes du marché.

Cet article s'organise en trois parties. La première partie présente les principales caractéristiques des modes de contrôle inter-organisationnels décrits dans la littérature et développe le contrôle par la confiance, ses fondements, implications et limites éventuelles. La deuxième partie présente l'étude de cas menée et décrit l'histoire de la rupture du contrôle par la confiance dans un tissu industriel local. La troisième partie présente les implications de cette rupture pour les parties et propose des éléments d'interprétation des fragilités du contrôle par la confiance. 


\section{Le contrôle par la confiance face aux autres modes de contrôle}

Après avoir présenté les modes de contrôle inter-organisationnel qui apparaissent de manière récurrente dans la littérature, nous nous concentrerons sur le contrôle par la confiance et nous en exposerons les fondements, les bienfaits ainsi que les fragilités potentielles.

\subsection{Les modes de contrôle client-fournisseur}

Les chercheurs en contrôle qui ont investi le champ des relations inter-organisationnelles ont largement intégré dans leurs travaux les apports de la littérature inter-organisationnelle (en particulier les travaux de l'approche transactionnelle et de l'approche de l'échange relationnel) et ceux de la littérature intra-organisationnelle (en particulier les travaux d'Ouchi, $1980^{2}$ ) pour proposer des modes de contrôle correspondant aux diverses situations d'échange (Dekker, 2004 ; Dumoulin, 1997 ; Dumoulin et Gbaka, 1997 ; Froehlicher, 2000 ; Hakansson et Lind, 2004 ; Langfield-Smith et Smith, 2003 ; van der Meer-Kooistra et Vosselman, 2000). Trois modes de contrôle sont récurrents dans ces recherches (Hakansson et Lind, 2004) : le marché, la bureaucratie et un troisième mode de contrôle appelé contrôle social, contrôle par la confiance ou clan.

Dans notre revue de littérature, nous regroupons le troisième mode de contrôle sous le terme de «contrôle par la confiance ». En effet, le clan et l'échange relationnel -fondé sur la confiance et les normes relationnelles- peuvent être considérés comme des concepts proches, selon Heide (1994), car ils reposent sur des normes selon lesquelles les objectifs individuels ne peuvent être atteints que par l'action jointe. En outre, le contrôle social est proche du clan car il implique une entente entre les acteurs sur les valeurs et les compétences qui fondent la coopération.

Le tableau 1, construit à partir de ces travaux ${ }^{3}$ présente une synthèse des trois modes de contrôle selon les dispositifs et les objets du contrôle (qui sont deux dimensions proposées par Chiapello (1996) pour analyser les modes de contrôle organisationnel). Nous présentons également les facteurs de contingence de chaque mode de contrôle. 
Tableau 1 : Trois modes de contrôle client-fournisseur et leurs facteurs de contingence

\begin{tabular}{|c|c|c|c|}
\hline & Contrôle par le marché & Contrôle bureaucratique $^{4}$ & Contrôle par la confiance \\
\hline $\begin{array}{l}\text { Dispositifs de } \\
\text { contrôle }\end{array}$ & 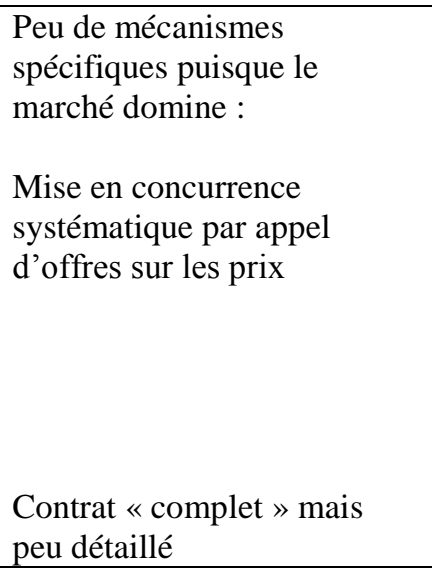 & $\begin{array}{l}\text { Le contrôle repose sur } \\
\text { l'autorité et les règles fixées } \\
\text { à l'avance ; critères de } \\
\text { sélection détaillés; } \\
\text { supervision directe ; revues } \\
\text { formelles } \\
\text { Contrats incomplets mais } \\
\text { détaillés }\end{array}$ & $\begin{array}{l}\text { Au départ, le contrôle repose } \\
\text { sur la réputation et les } \\
\text { expériences antérieures ; puis, } \\
\text { les contacts réguliers, } \\
\text { l'intensification des } \\
\text { communications et l'action } \\
\text { jointe stimulent la confiance } \\
\text { Contrats incomplets et peu } \\
\text { détaillés }\end{array}$ \\
\hline $\begin{array}{l}\text { Objets du } \\
\text { contrôle }\end{array}$ & $\begin{array}{l}\text { Les résultats (souvent prix, } \\
\text { qualité, délais) }\end{array}$ & $\begin{array}{l}\text { Les résultats et / ou les } \\
\text { comportements } \\
\text { (principalement les procédés } \\
\text { de travail) }\end{array}$ & $\begin{array}{l}\text { Les capacités et les } \\
\text { comportements relationnels }\end{array}$ \\
\hline $\begin{array}{l}\text { Facteurs de } \\
\text { contingence }\end{array}$ & $\begin{array}{l}\text { Sur les transactions: } \\
\text { Forte connaissance des } \\
\text { résultats } \\
\text { Forte répétitivité des } \\
\text { transactions } \\
\text { Faible spécificité des actifs } \\
\text { Sur l'environnement : } \\
\text { Beaucoup d'intervenants } \\
\text { substituables } \\
\text { Environnements dynamiques }\end{array}$ & $\begin{array}{l}\text { Sur les transactions: } \\
\text { Forte connaissance des } \\
\text { processus } \\
\text { Faible à moyenne } \\
\text { répétitivité des transactions } \\
\text { Actifs modérément } \\
\text { spécifiques } \\
\text { Sur l'environnement : } \\
\text { Risques du marché modérés } \\
\text { Contingences futures } \\
\text { connues } \\
\text { Environnements stables }\end{array}$ & $\begin{array}{l}\text { Sur les transactions: } \\
\text { Faible connaissance des } \\
\text { résultats et des processus } \\
\text { Faible répétitivité des } \\
\text { transactions } \\
\text { Actifs très spécifiques } \\
\text { Sur l'environnement: } \\
\text { Peu d'acteurs sur le marché } \\
\text { Incertitude sur les } \\
\text { contingences futures } \\
\text { Environnements stables }\end{array}$ \\
\hline
\end{tabular}

Parmi ces trois modes de contrôle, le contrôle par le marché et le contrôle bureaucratique s'appuient largement sur des dispositifs visibles de la coopération (par ex. le contrat, les règles, les résultats mesurables). Le contrôle par la confiance repose au contraire sur des mécanismes invisibles. Il apparaît souvent comme une alternative au prix et à l'autorité formelle lorsque ceux-ci s'avèrent inefficaces. Nous nous proposons d'expliciter la manière dont ce mode de contrôle se construit et opère dans les relations client-fournisseur.

\subsection{Le contrôle par la confiance : fondements, bienfaits et questionnements}

\subsubsection{Les caractéristiques du contrôle par la confiance}

La confiance est définie de bien des manières dans la littérature. Toutefois, on peut distinguer deux conceptions principales : la première considère que la confiance est une anticipation sur 
le fait que les attentes ne seront pas déçues (Zucker, 1986); la seconde, plus restreinte, suggère que la confiance est une croyance vis-à-vis d'autrui (Sako, 1992). Cette croyance concerne aussi bien les capacités de l'autre que sa volonté de bien faire. Dans cette perspective, avoir confiance en quelqu'un, c'est croire qu'il agira de façon positive chaque fois qu'il le peut (Guibert et Dupuy, 1997). C'est généralement à cette seconde conception que les auteurs qui s'intéressent au contrôle par la confiance se réfèrent. Sako (1992) distingue trois formes de confiance : la confiance contractuelle qui repose sur les standards moraux d'honnêteté et de respect de la parole donnée ; la confiance de compétence qui est liée à l'assurance que l'autre a les compétences requises pour assurer la prestation; et la confiance de bonne volonté qui est la croyance que l'autre n'entreprendra rien de négatif même s'il en a l'occasion et l'intérêt. Le contrôle par la confiance apparaît comme la résultante de la combinaison de ces trois formes qui évoluent et se renforcent dans le temps (van der MeerKooistra et Vosselman ; Langfield-Smith et Smith, 2003 ; Dekker, 2004).

La confiance ne naît pas d'emblée, mais elle se construit. Bien que la phase de sélection -ou phase de contact pour Larson (1992) ou van der Meer-Kooistra et Vosselman (2000)- soit souvent présentée comme étant le préliminaire de la relation, les partenaires potentiels ont souvent eu d'autres expériences ensemble. D'ailleurs les fournisseurs sont choisis sur un nouveau projet sur la base de leur réputation, éventuellement de leur qualification (selon des normes standardisées) -qui sont des «dispositifs de jugement » (Karpik, 1996) donnant à l'échange un «cadre relationnel » (Froehlicher, 2000)- et sur la base des interactions passées dans lesquelles ils se sont révélés dignes de confiance. Ainsi, le choix des fournisseurs est aussi bien déterminé par les caractéristiques des individus que par leur savoir-faire. Dans le contrôle par la confiance, s'il existe un contrat pour formaliser l'entente mutuelle, c'est un contrat cadre qui ne donne aucun détail sur le déroulement de la coopération. Il ne saurait être un mécanisme de contrôle. Larson (1992) décrit, à partir d'une étude empirique, les différentes étapes de construction du contrôle par la confiance. Dans les phases d'échange (phases d'essai puis d'intégration), les interactions répétées entre les individus permettent par des processus d'apprentissage et d'adaptation, l'émergence de règles explicites (procédures de communication, de partage des informations) et implicites (honnêteté, transparence, réciprocité, équité). Ces règles, qui sont des normes relationnelles comportementales, consolident la coopération et renforcent la confiance entre les parties.

Adapté aux relations qui demandent des actifs spécifiques, aux situations où les résultats et le processus de transformation sont peu connus et aux environnements incertains, le contrôle par 
la confiance se justifie dans les cas où les conflits d'intérêts sont faibles et où la congruence des buts -c'est-à-dire le partage des buts à long terme- est élevée (Dumoulin, 1997).

Si les ressorts de ce mode de contrôle sont, d'après les chercheurs, principalement informels, les liens entre contrôle formel et confiance exigent toutefois un développement particulier. Pour un grand nombre de chercheurs, la confiance est une alternative au contrôle formel (Larson, 1992 ; Ring et Van de Ven, 1992). S'appuyant sur les travaux de Macaulay (1963), certains indiquent que le contrôle formel détruit la confiance, au moins à partir d'un certain niveau de proximité relationnelle car il entraîne la suspicion (Dekker, 2004 ; Nooteboom et $a l .$, 1997). De même, Dumoulin et Gbaka (1997) indiquent que le contrôle se développe le long d'un continuum qui oppose contrôle total (par spécification des tâches) et confiance. L'étude empirique d'Andaleeb (1995) confirme d'ailleurs le fait que, plus le niveau de confiance entre les partenaires est élevé, moins le contrôle est utilisé. Gosse et al. (2002) suggèrent toutefois que la substitution du contrôle par la confiance au contrôle formel s'impose plus qu'elle n'est un choix lorsque le client ne sait évaluer la prestation de son fournisseur.

D'autres chercheurs considèrent au contraire que le contrôle informel et le contrôle formel sont complémentaires pour créer un «climat de confiance ${ }^{5}$ » entre les parties (Das et Teng, 1998 ; Guibert et Dupuy, 1997 ; Poppo et Zenger, 2002). La thèse défendue par ces auteurs est la suivante: l'élaboration et la mise en œuvre des mécanismes de contrôle formel (contrats, règles, systèmes de récompense) impliquent la multiplication des interactions entre les parties et ne sont efficaces que s'ils sont mis en perspective avec le contrôle informel qui émerge de ces interactions ; réciproquement, la connaissance partagée, facilitée par le contrôle informel, permet un raffinement des dispositifs formels. Contrôle formel et contrôle informel peuvent donc se développer ensemble de manière cohérente. Les résultats de l'étude de Dumoulin et al. (2000), qui rejettent l'hypothèse de la relation substitutive entre le contrat et la confiance, et de celle de Guibert et Dupuy (1997), qui n'identifient pas de lien entre la confiance et la formalisation contractuelle du contrôle, abondent dans ce sens. Dans cette perspective, les organisations qui privilégieraient uniquement les logiques informelles se priveraient des mécanismes formels qui sont tout autant des garants de la cohésion organisationnelle (Guibert et Dupuy, 1997). 


\subsubsection{Les implications de la confiance}

Les implications de la confiance sur la coopération ont largement été étudiées dans la littérature. Excepté les travaux sociologiques de Neuville (1997) qui indiquent que la confiance peut être une stratégie du fournisseur pour masquer sa défaillance et se comporter de manière opportuniste, la plupart des chercheurs prêtent à la confiance des propriétés vertueuses pour la coopération inter-entreprises.

En réduisant l'incertitude et les risques (Adler, 2001; Ring et Van de Ven, 1994), la confiance permet de développer des relations durables (Ganesan, 1994 ; Langfield-Smith et Smith, 2003; van der Meer-Kooistra et Vosselman, 2000), de favoriser l'engagement (Guibert, 1996) et de transformer des engagements problématiques en engagements crédibles (Karpik, 1996).

De plus, la confiance a un effet dissuasif sur les comportements opportunistes (Gulati, 1995 ; Larson, 1992). Elle permet également de mieux reconnaître de tels comportements lorsqu'ils surviennent car, nécessitant la multiplication des interactions pour se développer, elle se traduit par une diminution de l'asymétrie d'information (Saxton 1997). Ainsi, elle permet d'accroître la prévisibilité du partenaire (Langfield-Smith et Smith, 2003) et sa satisfaction (Ganesan, 1994).

En outre, «en remplaçant les contrats par des poignées de main » (Adler, 2001, p. 219), la confiance permet de réduire l'étendue des contrats (Brousseau, 1996 ; Larson, 1992), les risques d'agence et les coûts de transaction (Dyer et Chu, 2000 ; Gulati, 1995), ce qui améliore la compétitivité des partenaires (Noordewier et al. 1990) et plus largement leur performance (Aulakh et al., 1996 ; Poppo et Zenger, 2002).

Enfin, la confiance a également un effet positif sur le déroulement de la coopération. Elle réduit les problèmes de coordination (Adler, 2001), permet d'outrepasser les inégalités de court terme (Guibert, 1999), active l'interaction entre les parties (van der Meer-Kooistra et Vosselman, 2000), accroît leurs efforts joints (Dyer et Chu, 2000) et facilite la résolution des conflits (Das et Teng, 1998; Ring et Van de Ven, 1994). Les routines de partage d'informations sont plus efficientes (Dyer et Chu, 2000 ; Langfield-Smith et Smith, 2003). La confiance favorise également la flexibilité (Dyer et Chu, 2000 ; Ring et Van de Ven, 1992), le 
développement de la connaissance (Adler, 2001), l'apprentissage (Guibert, 1999) et plus largement l'adaptation inter-firmes (Larson, 1992).

\subsubsection{La confiance en question}

Bien que la plupart des chercheurs indiquent que la confiance implique une prise de risque, ceux-ci s'attachent bien plus à développer le processus de construction de la confiance et ses bienfaits qu'à présenter les fragilités de ses fondements et à en développer les implications si l'un des partenaires fait défaut.

Le principal risque pour celui qui fait confiance est bien sûr de voir sa confiance trahie. Toutefois, ce risque est limité si l'on considère que les relations sont encastrées dans un réseau social. Comme le souligne Granovetter (1985, p. 490), «l'argument de l'encastrement met l'accent sur le rôle des relations personnelles et des structures (ou réseaux) de telles relations pour générer la confiance et décourager la malfaisance ». Les sanctions sociales qui sont un rempart contre l'opportunisme peuvent aller de l'atteinte à la réputation jusqu'à l'exclusion de la communauté (Dyer et Chu, 2000). Cet argument reste valable tant que les partenaires sont sensibles à la sanction sociale, mais s'ils changent de réseau ou si l'intérêt économique à faire défaut devient trop important, l'étude de Wathne et al. (2001) montre que le client n'hésite pas à changer de partenaire.

Par ailleurs, les auteurs mettent en avant le fait que la confiance est adaptée aux situations où les parties peuvent s'entendre sur un objectif commun à long terme -c'est-à-dire aux situations où la congruence des buts est forte (Dumoulin 1997). En effet, comme le soulignent Dwyer et al. (1987), maintenir une relation de coopération exige l'emploi de ressources considérables (matérielles mais également psychologiques); et, plus les intérêts sont divergents, plus le coût de la coopération est élevé. Toutefois, fonder le contrôle par la confiance sur la congruence des buts est d'une grande fragilité si, à l'instar de Crozier et Friedberg (1977), on considère que les buts des individus (et des organisations) sont divers et changeants.

Enfin, selon Dyer et Chu (2000), la confiance ne peut être conceptuellement placée que d'un individu envers un autre individu (ou groupe d'individus), et non d'une organisation vers une autre organisation. Lorsque les individus d'une même organisation partagent une 
«orientation collective de confiance vis-à-vis des individus de la firme partenaire », Zaheer et al. (1998, p. 143) indiquent qu'on peut parler de confiance inter-organisationnelle. Toutefois, ces auteurs ne détaillent pas les processus qui permettent de développer cette orientation collective. Or, d'après Guibert (1999), la confiance ne se transmet pas spontanément du niveau inter-individuel au niveau inter-organisationnel. Dès lors, un changement d'individus risque d'anéantir la confiance entre les entreprises (Nooteboom et al. 1997), ce qui fragilise considérablement les fondements du contrôle par la confiance.

Nous avons présenté certaines fragilités de la confiance qui peuvent être proposées a priori d'après la littérature. En ce qui concerne les implications de la rupture d'un lien de confiance, les travaux empiriques sont peu nombreux. Dwyer et al. (1987), pour qui la confiance est centrale dans les différentes phases de la coopération proposent une dernière phase qu'ils appellent «dissolution ». Lorsque l'une des parties évalue ses insatisfactions supérieures aux bénéfices qu'elle retire de la coopération, un processus de désengagement s'opère. D'après ces auteurs, ce processus est mal expliqué, mais il génère un stress émotionnel et physique significatif.

Dès lors, il paraît pertinent d'étudier le processus de rupture de relations de confiance, d'en comprendre les causes et les implications pour les deux parties. Cette analyse nous amènera à approfondir la connaissance empirique des fragilités du contrôle par la confiance.

\section{La rupture d'un mode de contrôle par la confiance dans les relations client-fournisseur de la vallée de la Maurienne}

Dans cette partie, nous présentons la méthodologie retenue. Puis nous caractérisons le contrôle par la confiance qui prévalait jusqu'au milieu des années 1990 entre clients et fournisseurs d'entretien et de maintenance de la vallée de la Maurienne. Enfin, nous expliquons l'évolution des pratiques d'achat des clients qui ont impliqué la rupture de ce mode de contrôle. 


\subsection{Méthodologie de l'étude des relations client-fournisseur dans le bassin de la Maurienne}

Cette recherche s'appuie sur une étude de cas des relations entre des clients et leurs fournisseurs d'entretien et de maintenance dans la vallée de la Maurienne ${ }^{6}$, dans la mesure où, dans ce bassin, les relations client-fournisseur ont été confrontées à des mutations profondes dans les années 1990. En effet, les relations de proximité et de confiance ont été mises en porte-à-faux par l'introduction de nouvelles pratiques d'achat et de contrôle de la part des clients, eux-mêmes soumis à de nouvelles contraintes.

La méthodologie retenue est qualitative et exploratoire. Elle vise à explorer un processus de rupture d'un contrôle par la confiance dans des relations client-fournisseur. Les données présentées ici ne concernent qu'un groupe d'entreprises dans un bassin géographique limité. Bien que l'utilisation d'un seul cas à des fins descriptives et théoriques soit controversée, Chakravarthy et Gargiulo (1998) recommandent cette méthode en ce qui concerne l'étude des dynamiques de changement et suggèrent de décrire l'objet du changement et le contexte dans lequel il s'opère.

Dans la vallée de la Maurienne, nous avons recensé six sites industriels clients et quarante fournisseurs locaux dont onze fournisseurs d'entretien et de maintenance. Pour cette étude, nous avons mobilisé toutes les sources d'information proposées par Yin (1989) à l'exception de l'observation participante et de la simulation. Nous avons interrogé à plusieurs reprises (dans un souci d'approfondissement et de validation) l'ensemble des directeurs des sites des entreprises clientes et les dirigeants des onze entreprises fournisseurs. Nous avons également interrogé les acteurs locaux de la vallée (maires, responsables d'agences publiques locales, etc.). En outre, nous avons analysé et synthétisé une large documentation interne et externe : pour les entreprises clientes, les tableaux de bord de suivi des frais externes, les tableaux de bord d'activité, les documents explicitant la politique d'achat; pour les fournisseurs, nous avons obtenu des données sur l'activité et leurs résultats à partir d'une consultation de la base de données de la Banque de France.

Les données ont été analysées selon la méthode préconisée par Miles et Huberman (1991) en construisant des catégories pertinentes a priori pour notre thème de recherche à partir d'une analyse de la littérature, catégories que nous avons complétées grâce aux données du terrain. 


\subsection{A l'origine, un mode de contrôle par la confiance}

\subsubsection{La nature des transactions : des tâches spécifiques, peu répétitives, de proximité}

Jusqu'au milieu des années 1990, les six établissements industriels clients implantés dans la vallée (dans les secteurs de la métallurgie, de la sidérurgie, de la chimie et de l'hydraulique) ${ }^{7}$ confiaient à un tissu de petites entreprises locales des travaux de maintenance et d'entretien des équipements (plus rarement des travaux neufs).

Historiquement, ces $\mathrm{PME}^{8}$ ont été créées lorsque les clients avaient décidé d'externaliser leurs tâches de maintenance (préventive et curative) des équipements industriels. De ce fait, les fournisseurs, dédiés à ces entreprises, se sont organisés pour pouvoir répondre à tout moment aux besoins de leurs clients (proximité géographique, mise à disposition de salariés, etc.). Ils se sont spécialisés dans un domaine particulier, voire dans l'entretien et la maintenance d'un équipement particulier. Ils ont ainsi développé des compétences spécifiques, gage de la qualité et de la rapidité de leurs interventions. Dès lors, les clients ont progressivement abandonné la maîtrise de ces compétences et se sont reposés sur un petit nombre de fournisseurs spécialisés.

"On est situé dans une vallée encaissée : il fallait des prestataires sur place pour intervenir rapidement " [Directeur de l'établissement client T].

«Ils étaient devenus de vrais Zorros. On leur demandait une entière disponibilité; on leur demandait d'être corvéables, et on était prêt à payer pour cela». [Directeur de l'établissement industriel client P].

"On connaît bien leurs installations, avec leurs spécificités et leurs risques. Dans la plupart des cas, on règle les problèmes avant même qu'ils s'en soient rendus compte » [Directeur de l'entreprise fournisseur C].

«Ils ont chacun leur spécialité, avec peu de concurrence locale» [Directeur de l'établissement client M]

Ainsi, les clients ont progressivement perdu leur connaissance du processus de réalisation des tâches qui, de surcroît, sont peu répétitives (en particulier, la maintenance curative se caractérise par des aléas et ne peut strictement pas répondre à un cahier des charges prédéfini). Dans ce cas, les caractéristiques des transactions (compétences spécifiques requises, faible répétitivité, faible connaissance du processus de transformation) et de l'environnement (peu d'acteurs spécialistes sur chaque équipement) sont cohérents, d'après la littérature, avec un recours à un contrôle par la confiance qui a effectivement été privilégié pendant longtemps. 


\subsubsection{Dispositifs et objets du contrôle : des relations durables pour un «travail bien fait»}

Jusqu'au milieu des années quatre-vingt dix, les établissements clients confiaient les marchés aux gens du crû, ceux qu'ils connaissaient et qui les connaissaient, ceux en qui ils avaient confiance. Ainsi, la sélection était fondée sur la réputation des fournisseurs concernant la qualité de leur prestation et sur une longue expérience de coopérations antérieures. Les critères du «travail bien fait » et de la disponibilité étaient prépondérants dans les choix car ils permettaient d'anticiper les situations critiques et d'assurer la continuité de l'exploitation industrielle. En outre, les salariés des établissements clients et des fournisseurs étaient inscrits dans les mêmes réseaux sociaux : les relations inter-entreprises étaient enchevêtrées dans des relations inter-personnelles de nature privée.

«Le responsable du service de maintenance de l'entreprise $P$, c'est le voisin du frère du directeur de l'entreprise $B »$ [Directeur de l'entreprise fournisseur L].

"Le gérant de l'entreprise C, je le connais bien car il est du même village; je sais que je n'aurai jamais de problème avec lui » [Directeur de l'établissement client O].

"Ils nous connaissent bien et on travaille bien ensemble» [Directeur de l'établissement client T].

«Avec ceux des fournisseurs que je connais, je suis sûr et tranquille. » [Directeur de l'établissement P]

Du point de vue de la formalisation de la coopération, les contrats établis entre les clients et les fournisseurs étaient de différentes natures, selon le type et le montant de la prestation : des devis validés par les responsables locaux des établissements clients pour les travaux neufs à partir d'une demande exprimée verbalement sur le site industriel, des forfaits pour l'entretien à l'année dans le cadre de contrats reconduits automatiquement, ou une facturation au temps passé pour la maintenance curative. Dans tous les cas, les contrats n'étaient pas assez précis et détaillés (en termes de cahier des charges et de systèmes de récompense) pour servir de dispositifs de contrôle. En outre, cette forme lâche de contractualisation permettait de régler les éventuels conflits de manière harmonieuse : par exemple, quand le temps passé sur le chantier dépassait le devis initial, le chef de service de l'entreprise cliente s'arrangeait pour passer le contrat suivant au même fournisseur.

"Pour la maintenance, on travaillait sans devis préalable; on facturait tous les mois le travail effectué et cela rentrait dans leur budget. En fin d'année, s'ils [les établissements industriels] avaient des problèmes de budgets, on reportait un peu nos factures sur l'année suivante. Pour les grands travaux, on s'était mis d'accord sur un forfait. Avec le forfait, on peut toujours tomber sur un os; alors, ils comprenaient et pour nous récupérer, ils nous donnaient un nouveau marché de travaux » [Directeur de l'entreprise fournisseur B]. 
Les liens informels de proximité étaient ainsi déterminants dans les modes de coordination entre les entreprises, qui, selon les responsables d'entreprises rencontrés, obéissaient à des «échanges de bons procédés » où «tout le monde y trouvait son compte » [responsable du comité de développement local] et qui permettaient, selon les termes de Raveyre et Saglio (1984), de «moduler les effets de la concurrence, d'organiser les confrontations et les conflits ». Les conflits étaient de toute façon assez rares, les clients et les fournisseurs partageant les mêmes intérêts (obtenir / délivrer une prestation de qualité) dans un contexte où le prix passait au second plan.

En définitive, les relations entre clients et fournisseurs reposaient largement sur la confiance (à la fois contractuelle, de bienveillance et de compétence) et sur les normes relationnelles (de solidarité, de continuité, de réciprocité et de fonctionnalité du conflit). Ce mode relationnel permettait d'inscrire la coopération dans la continuité, chacun considérant le fonctionnement d'ensemble comme étant de qualité.

\subsection{L'évolution des pratiques d'achat et de contrôle des clients.}

A partir du milieu des années 1990, les établissements des grands groupes industriels sont soumis à des contraintes de rationalisation des coûts et, dans le même temps, les directions nationales décident de centraliser les décisions d'achat. Ces évolutions sont à rapprocher de pratiques de grands groupes qui se sont développées à partir des années quatre-vingts, ces derniers ayant tout à la fois rationalisé et centralisé les processus d'achat (Gorgeu et Mathieu, 1983).

Face à la crise qu'ils connaissent à ce moment-là, les établissements des grands groupes industriels étudiés partent, selon un des directeurs d'usines, «dans une chasse au gaspi » et tous revoient à la baisse leurs volumes de sous-traitance de maintenance et d'entretien (à hauteur de $15 \%$ à $30 \%$ selon les sites).

«Si on veut être maintenu dans le groupe, on doit en permanence apporter la preuve de notre existence, et continuer d'accroître la productivité. On a revu ligne par ligne les budgets de fonctionnement hors frais de personnel, et on a réduit la maintenance car c'est facile à réduire » [Directeur de l'établissement client T].

A cette évolution, en partie conjoncturelle, vient s'ajouter un mouvement de fond qui a évincé certaines entreprises locales de la relation d'échange : l'établissement de processus centralisés de sélection des fournisseurs. Par ce mouvement de centralisation, la décision d'achat, y 
compris celle concernant la maintenance, est en grande partie remontée au niveau des directions des groupes. Les directions locales ont alors perdu leur marge d'autonomie en la matière. Au-delà de la délégation de signature dont le plafond a été fortement abaissé, les directions locales doivent lancer des appels d'offres auprès d'un volant élargi d'entreprises pré-sélectionnées par la direction nationale des achats dans un panel, là où les contrats étaient antérieurement reconduits tacitement avec les fournisseurs locaux.

"On a de nouvelles directives du groupe sur la sélection des fournisseurs : ils nous poussent à changer de prestataire; les choses sont reprises au niveau du groupe qui a le sentiment que les choses ont été mal faites » [Directeur de l'établissement client $O]$.

"Nous avons l'obligation de consulter le panel de fournisseurs homologués lors d'appels d'offres. Quand un homologué arrive en tête ou à égalité, on a l'obligation de le prendre. S'il est homologué, on ne peut pas invoquer la qualité de travail d'un autre pour ne pas le prendre. Cela se passe à la direction des achats du groupe, avec des antennes d'achat locales. " [Directeur de l'établissement client P]

Le processus de sélection des fournisseurs habilités à travailler pour l'entreprise (ceux qui sont intégrés dans le panel) s'est donc déplacé au niveau national de l'entreprise cliente, avec simultanément une modification dans les critères de sélection : ils s'avèrent être plus normés, fondés essentiellement sur les prix.

«La concurrence joue maintenant sur les prix : on n'a pas de cahiers des charges précis et il est difficile d'établir précisément les critères qualité. Tout est sur les prix et l'argument de la proximité géographique tombe " [Directeur de l'établissement client T].

"'Elle nous connaît bien', ou 'on sait qu'ils font du bon boulot', c'est un argument qui ne passe pas auprès de la direction des achats » [Directeur de l'établissement client M]

"Quand on les met en concurrence, on entre dans une logique de moins-disant» [Directeur de l'établissement client $O$ ].

On passe ainsi d'un fonctionnement de marché quasi de gré à gré, entre personnes se connaissant bien et s'évaluant à l'aune de ces relations établies, à l'établissement de contrats issus de procédures d'appels d'offre avec de nouveaux fournisseurs sur la base de résultats (en premier lieu, les prix). Les dispositifs et les objets du contrôle réalisé par les clients sont ainsi caractéristiques d'un mode de contrôle par le marché, alors que, dans le même temps, les caractéristiques des transactions n'ont pas évolué. Le tableau 2 propose une synthèse de l'évolution du mode de contrôle dans les relations client-fournisseur de la vallée de la Maurienne. 
Tableau 2 : Synthèse de l'évolution des relations client-fournisseur dans la vallée de la Maurienne

\begin{tabular}{|c|l|l|}
\hline \multicolumn{3}{|c|}{ Nature de la relation et du mode de contrôle } \\
\hline & \multicolumn{1}{|c|}{ Avant } & \multicolumn{1}{c|}{ Après } \\
\hline Mode de contrôle & Contrôle par la confiance & Contrôle par le marché \\
\hline Objets du contrôle & $\begin{array}{l}\text { Les compétences des fournisseurs, la qualité de } \\
\text { la prestation (travail bien fait) et du service } \\
\text { associé (réactivité, disponibilité) } \\
\text { Pas de critères formalisés }\end{array}$ & Les résultats et en premier lieu les prix \\
\hline $\begin{array}{c}\text { Nature des relations } \\
\text { inter-entreprises }\end{array}$ & $\begin{array}{l}\text { Relations interindividuelles locales, entre un } \\
\text { directeur de PME et des directeurs de sites }\end{array}$ & $\begin{array}{l}\text { Relations de marché globales, gérées par les } \\
\text { directions centrales des achats des groupes } \\
\text { industriels }\end{array}$ \\
\hline $\begin{array}{c}\text { Champ de mise en } \\
\text { concurrence }\end{array}$ & Sollicitation des prestataires locaux uniquement & $\begin{array}{l}\text { Extension des sollicitations des clients à des } \\
\text { prestataires régionaux voire nationaux }\end{array}$ \\
\hline $\begin{array}{c}\text { Critères et processus } \\
\text { de sélection des } \\
\text { fournisseurs }\end{array}$ & $\begin{array}{l}\text { Réputation et expériences communes passées ; } \\
\text { reconduction tacite des contrats et facturation } a \\
\text { posteriori sur la base des réalisations } \\
\text { (maintenance) ou de « forfaits » (travaux neufs) }\end{array}$ & $\begin{array}{l}\text { Mise en concurrence des fournisseurs par des } \\
\text { procédures d'appels d'offres sur la base de } \\
\text { cahiers des charges spécifiés à l'avance }\end{array}$ \\
\hline $\begin{array}{c}\text { Cadre contractuel } \\
\text { Contrat-cadre ou pas de contrat : ajustements } \\
\text { mutuels en cas de défauts de réalisation ou en } \\
\text { cas de dépassement d'activités }\end{array}$ & $\begin{array}{l}\text { Application stricte des clauses contractuelles ; les } \\
\text { fournisseurs sont rémunérés sur la base de } \\
\text { l'atteinte des résultats spécifiés au contrat }\end{array}$ \\
\hline
\end{tabular}

\section{Les impacts de la rupture du mode de contrôle par la confiance sur les fournisseurs et les clients locaux}

Nous avons cherché à identifier les implications de la rupture du contrôle par la confiance aussi bien pour les fournisseurs que pour les établissements clients locaux. Le tableau 3 présente une comparaison de la situation des partenaires avant et après cette rupture. 
Tableau 3 : Synthèse de la situation des fournisseurs locaux et des clients locaux avant et après la rupture du mode de contrôle par la confiance

\begin{tabular}{|c|c|c|}
\hline \multicolumn{3}{|c|}{ Situation des fournisseurs locaux } \\
\hline & Avant & Après \\
\hline Evolution de l'activité & $\begin{array}{l}\text { Un chiffre d'affaires annuel moyen de } \\
\text { prestations confiées localement estimé à } 250 \mathrm{MF}\end{array}$ & $\begin{array}{l}\text { Chute de } 20 \% \text { à } 75 \% \text { de l'activité des } \\
\text { fournisseurs locaux }\end{array}$ \\
\hline $\begin{array}{c}\text { Temporalité des } \\
\text { contrats }\end{array}$ & $\begin{array}{l}\text { Des prestations pluri-annuelles reconduites } \\
\text { automatiquement }\end{array}$ & Des contrats morcelés \\
\hline Types de prestations & $\begin{array}{l}\text { Entretien complet d'équipements et travaux } \\
\text { neufs }\end{array}$ & Entretien partiel des équipements \\
\hline $\begin{array}{c}\text { Visibilité sur le carnet } \\
\text { de commandes }\end{array}$ & $\begin{array}{l}\text { Visibilité moyenne des prestataires locaux sur } \\
\text { leur carnet de commandes évaluée à } 12 \text { mois }\end{array}$ & $\begin{array}{l}\text { Visibilité moyenne des prestataires locaux } \\
\text { évaluée à } 1-6 \text { mois }\end{array}$ \\
\hline Evolution des effectifs & $\begin{array}{l}\text { Effectifs des entreprises prestataires stables, } \\
\text { avec un seuil de } 50 \text { salariés }\end{array}$ & $\begin{array}{l}\text { Réduction des effectifs chez les prestataires } \\
\text { locaux; recours accru à la flexibilité externe de } \\
\text { l'emploi }\end{array}$ \\
\hline $\begin{array}{c}\text { Pérennité des } \\
\text { fournisseurs locaux }\end{array}$ & $\begin{array}{l}\text { Des entreprises créées de longue date, a priori } \\
\text { pérennes }\end{array}$ & $\begin{array}{l}\text { Quelques cas de dépôt de bilan, craintes générales } \\
\text { sur la survie des fournisseurs locaux }\end{array}$ \\
\hline \multicolumn{3}{|c|}{ Situation des établissements clients locaux } \\
\hline & Avant & Après \\
\hline $\begin{array}{l}\text { Coût direct des } \\
\text { prestations de } \\
\text { maintenance }\end{array}$ & $\begin{array}{l}\text { Un « surcoût » des prestations locales estimé } a \\
\text { posteriori à } 30 \%\end{array}$ & $\begin{array}{l}\text { Une diminution des budgets de maintenance des } \\
\text { clients de l'ordre de } 20 \% \text { à } 40 \%\end{array}$ \\
\hline $\begin{array}{l}\text { Délais de réalisation } \\
\text { des prestations }\end{array}$ & $\begin{array}{l}\text { Réalisation immédiate des prestations de } \\
\text { maintenance et des travaux neufs ; forte } \\
\text { flexibilité opérationnelle des fournisseurs }\end{array}$ & $\begin{array}{l}\text { Allongement des délais moyens de réalisation des } \\
\text { prestations de maintenance et des travaux neufs ; } \\
\text { perte en flexibilité opérationnelle }\end{array}$ \\
\hline $\begin{array}{l}\text { Maîtrise des } \\
\text { compétences } \\
\text { spécifiques }\end{array}$ & $\begin{array}{l}\text { Compétences spécifiques liées à la maintenance } \\
\text { des équipements développées et maîtrisées par } \\
\text { les fournisseurs locaux }\end{array}$ & $\begin{array}{l}\text { Perte de maîtrise des compétences spécifiques } \\
\text { liées à la maintenance des équipements, les } \\
\text { nouveaux fournisseurs n'ayant pas développé ces } \\
\text { compétences }\end{array}$ \\
\hline $\begin{array}{l}\text { Coordination } \\
\text { opérationnelle avec le } \\
\text { fournisseur }\end{array}$ & $\begin{array}{l}\text { Coordination immédiate avec des fournisseurs } \\
\text { qui « connaissent bien » les équipements }\end{array}$ & $\begin{array}{l}\text { Investissement répété dans la relation avec des } \\
\text { nouveaux fournisseurs qui « connaissent mal » } \\
\text { les équipements }\end{array}$ \\
\hline Tâches des salariés & $\begin{array}{l}\text { Spécialisation et concentration des salariés sur } \\
\text { les tâches de « cœur de métier » }\end{array}$ & $\begin{array}{l}\text { Dispersion des salariés sur de nouvelles tâches, } \\
\text { avec des risques de mauvaise exécution des } \\
\text { tâches de « cœur de métier » }\end{array}$ \\
\hline $\begin{array}{l}\text { Gestion de l'emploi et } \\
\text { des compétences }\end{array}$ & $\begin{array}{l}\text { Bonne adéquation entre les compétences internes } \\
\text { et les besoins de l'organisation }\end{array}$ & $\begin{array}{l}\text { Nécessité de développer de nouvelles } \\
\text { compétences internes et d'organiser des mobilités } \\
\text { internes pour accompagner la réorganisation liée } \\
\text { aux nouvelles pratiques d'achat }\end{array}$ \\
\hline $\begin{array}{l}\text { Continuité de } \\
\text { l'exploitation } \\
\end{array}$ & Bonne continuité de l'exploitation & \begin{tabular}{|l|} 
Accroissement des situations d'urgence et de \\
dysfonctionnements dans l'exploitation
\end{tabular} \\
\hline $\begin{array}{l}\text { Etat général des } \\
\text { équipements }\end{array}$ & $\begin{array}{l}\text { Etat général des équipements jugé bon } \\
\text { Diminution régulière des délais d'arrêts partiels } \\
\text { des équipements }\end{array}$ & $\begin{array}{l}\text { Dégradation de l'état général des équipements ; } \\
\text { augmentation des délais d'arrêts partiels des } \\
\text { équipements }\end{array}$ \\
\hline
\end{tabular}

\subsection{Des fournisseurs locaux ébranlés}

L'ensemble de ces phénomènes s'est traduit chez les fournisseurs locaux par une diminution de leur activité allant de $20 \%$ à $75 \%$. En outre, l'activité qu'ils conservent est plus précaire, ce qui les a conduits à modifier les modalités d'emploi de leurs salariés. 


\subsubsection{Fragilisation de l'activité et de l'emploi chez les fournisseurs locaux}

Les fournisseurs locaux ont perdu une partie de leurs contrats antérieurs. En effet, les clients ont soit rapatrié, soit abandonné la réalisation de certaines tâches de maintenance. Ils ont également transféré certains contrats antérieurement confiés aux fournisseurs locaux à de nouveaux fournisseurs situés en dehors de la vallée.

«Au lieu de nous faire venir trois fois par semaine, on n'y va plus qu'une fois » [Directeur de l'entreprise fournisseur E]

"J'ai perdu le contrat et ils l'ont passé à une entreprise de Grenoble » [Directeur de l'entreprise fournisseur I]

En outre, la nature des contrats qu'ils conservent a changé : le prix moyen des prestations a diminué ; le nombre de contrats d'entretien systématiquement reconduits d'une année sur l'autre a drastiquement diminué ; et les fournisseurs doivent s'investir dans la réalisation de réponse à des appels d'offres dont l'issue est incertaine là où antérieurement cette tâche était quasi-inexistante.

"Pour rester dans la course, on a dû baisser nos prix d'au moins $10 \%$ et on n'a plus d'exclusivité nulle part » [Directeur de l'entreprise fournisseur B].

"Maintenant, je passe un temps fou à préparer des devis, des dossiers pour leurs appels, pour finalement obtenir moins de contrats » [Directeur de l'entreprise fournisseur D].

Cette nouvelle pression marchande se caractérise alors pour ces PME par un raccourcissement de la visibilité sur le carnet de commandes, par une flexibilisation des contrats passés, par un allongement des délais de décision des clients et par une réduction des volumes moyens d'activité par contrat.

«Cela fait dix-huit mois que je n'ai jamais plus de trois semaines de boulot devant moi » [Directeur de l'entreprise fournisseur D].

"Avant, on avait en charge l'entretien complet d'un équipement, maintenant on n'a plus que des petits bouts ». [Directeur de l'entreprise fournisseur G].

L'évolution des pratiques d'achat des clients s'est traduite par des réductions d'effectifs dans les PME concernées (en moyenne, diminution d'un tiers de l'effectif) et par le recours accru à des formes de flexibilité externe de l'emploi, avec un recours particulier à l'intérim.

\subsubsection{Tentatives de reconversion, impacts sur le bassin et sentiment d'abandon}

Devant cette nouvelle situation, quelques dirigeants des PME fournisseurs se sont lancés dans une recherche de diversification de leurs prestations et / ou de leurs clients. Ils ont engagé leur 
entreprise dans des processus de certification qualité (ISO 9000) et de rationalisation des coûts. Certains ont tenté de se regrouper pour répondre aux appels d'offres, mais n'ont pas réussi à obtenir les contrats. Les autres PME rencontrent des difficultés à faire le deuil de leurs clients traditionnels et à se reconvertir, soit parce qu'elles n'en ont pas les moyens, soit parce que leurs dirigeants y ont plus ou moins renoncé. La négation des critères qui prévalaient auparavant est très mal vécue par tous les responsables des PME locales. La rupture du lien commercial prend une dimension affective : certains dirigeants d'entreprises fournisseurs font état de situations de stress émotionnel, de lassitude, et de sentiment d'abandon.

«J'ai toujours bien fait mon boulot; nous, on est là et maintenant ils donnent le travail à d'autres » [Directeur de l'entreprise fournisseur J].

«De toute façon, on est trop petit pour passer dans leurs grilles » [Directeur de l'entreprise fournisseur A].

"Pour moi, c'est trop tard et je vais juste essayer de maintenir le cap sans trop de dégâts » [Directeur de l'entreprise fournisseur J].

"Et dire que j'ai travaillé 25 ans pour en arriver là ... » [Directeur de l'entreprise fournisseur B].

Enfin, cette décision de rationalisation-centralisation des achats s'est traduite par un déficit d'image locale des sites industriels, le sentiment de «trahison » étant repris et véhiculé non seulement par les fournisseurs locaux, mais aussi par les acteurs institutionnels locaux (maires, responsables de comité de développement local) que nous avons rencontrés. Elle s'est traduite par de nombreuses suppressions d'emplois dans les PME concernées, voire par des dépôts de bilan. La perte d'emplois directs dans les PME locales a été évaluée par le comité de développement local à environ cent emplois, et la perte d'emplois induits à environ deux cents emplois. Ce déficit d'emploi a été régulé par des migrations à l'extérieur de la vallée, accentuant le risque de dépeuplement de la vallée.

De ce fait, cette rupture de la relation de confiance a été interprétée localement comme une marque de désaffection des grands groupes industriels par rapport à un territoire qui s'est développé avec eux.

\subsection{Les effets induits pour les établissements clients locaux}

Pour les clients locaux, si le gain financier direct du changement de processus d'achat paraît évident puisque les clients ont obtenu une réduction des coûts d'achat de la maintenance et de l'entretien d'environ 30\%, l'introduction de ces nouvelles procédures d'achat comporte 
néanmoins des effets induits qui ont été constamment soulignés par les responsables locaux des établissements industriels clients sans qu'ils aient été pour autant évalués. On peut ainsi estimer que les conséquences organisationnelles du changement de prestataires ont été sousestimées, laissant penser à un postulat initial de type «l'intendance suivra » (Berry, 1983). Or, justement, l'intendance de cette décision semble lourde à gérer pour les usines et se traduit par un accroissement des dysfonctionnements organisationnels chez les clients.

\subsubsection{La perte d'un " slack organisationnel » comporte des effets induits sur la flexibilité opérationnelle}

Il apparaît que la proximité des relations client-fournisseur permettait de pallier des dysfonctionnements ou des carences organisationnelles des sites de production des clients, les fournisseurs remplissant une fonction de «slack organisationnel» ou encore d'amortisseur des situations critiques telles qu'une panne ou une surchauffe d'activité. Et, d'une certaine façon, cette contribution à la continuité de l'exploitation des entreprises clientes n'était pas directement facturée mais faisait partie de la relation. Ce modèle d'organisation, en lien étroit avec les entreprises de maintenance, était profondément ancré et assurait selon nos interlocuteurs, de bonnes conditions d'exploitation des sites.

A l'inverse, la rupture de ces relations client-fournisseur s'est traduite par un accroissement des situations d'urgence et par une prise de risque accrue en termes de continuité des exploitations industrielles et de dégradation des équipements. Dans les sites de métallurgie, il ressort par exemple un allongement des délais de maintenance curative, se traduisant par un allongement des délais moyens d'arrêts d'activités partiels. Les directeurs d'usines et les responsables d'ateliers des sites industriels concernés ont également tous affirmé que le transfert de l'activité de sous-traitance de maintenance auprès de fournisseurs situés en dehors de la vallée leur avait fait perdre en réactivité, notamment dans le cas de maintenance curative. De fait, les nouveaux fournisseurs sont géographiquement plus éloignés, programment avec le client le planning des visites, et les événements imprévus semblent donner lieu à une gestion plus compliquée et se traduisent par des temps d'arrêt plus longs.

"Quand un chef de service veut faire des travaux, le temps qu'il obtienne la signature, c'est devenu urgent » [Responsable industriel de l'établissement client P]. "Aujourd'hui, la direction peut refuser un prestataire quitte à retarder un contrat. Résultat, il y a plein de choses que l'on ne fait plus et on prend des risques pour l'exploitation » [Directeur de l'établissement client T]. 
"Maintenant, quand on a un problème avec une machine, on doit attendre que l'entreprise de maintenance arrive et on perd plus de temps qu'avant » [Directeur de l'établissement client A].

Or, comme le soulignent Van der Meer-Kooistra et Vosselman (2000), sous-traiter les activités de maintenance n'apporte un avantage économique à long terme que si la continuité de l'exploitation est assurée par la qualité des prestations des fournisseurs, et, selon eux, cette qualité ne peut provenir que des relations encastrées de proximité entre les acteurs. Dans le cas étudié, on peut estimer que les sites industriels des clients ont ainsi perdu en «flexibilité opérationnelle », et par conséquent en capacité d'adaptation des processus industriels et de l'organisation aux aléas (Reix, 1997).

\subsubsection{Une perte en compétences spécifiques et coûts de réorganisation}

Les mouvements simultanés de rapatriement d'activités de sous-traitance et de changement de prestataires ont introduit des difficultés en termes de gestion de l'emploi et des compétences. En effet, les clients s'étaient dépossédés des compétences spécifiques liées aux activités de maintenance acquises au fil du temps par les fournisseurs.

Le changement introduit dans les pratiques d'achat a ainsi impliqué une véritable réorganisation des sites industriels qui n'avait pas été anticipée : il a notamment fallu gérer des inadéquations entre les qualifications des salariés et les tâches rapatriées qui exigeaient des nouveaux besoins en compétences. Cette situation s'est traduite par un accroissement des coûts de formation, par des coûts de gestion des mobilités internes et par des coûts liés à l'abandon de certaines tâches que les salariés des entreprises clientes n'ont plus le temps d'assurer. Au-delà de ces coûts induits, les directeurs des établissements clients estiment que les salariés ne sont plus en mesure d'assurer pleinement leur cœur de métier, et qu'ils prennent des risques en termes de qualité et de continuité de l'exploitation.

"Pour nos salariés, ils doivent faire plus de choses qu'avant. En rapatriant, on a dî augmenter leur polyvalence et il a fallu investir en formation »[Directeur de l'établissement client T].

"Cela a été un vrai casse-tête : on était en sous-effectif, avec des tâches à rapatrier, et toute l'organisation a été chamboulée. " [Directeur de l'établissement client P].

"On en arrive à abandonner le travail de haut niveau pour régler de multiples petits problèmes et l'urgence s'installe » [Directeur de l'établissement client O].

En outre, le changement de prestataire implique un temps et des coûts d'adaptation du nouveau fournisseur liés à l'apprentissage des situations et des compétences spécifiques ; or, 
il apparaît que la distance géographique ne favorise pas cette acquisition de façon rapide et pérenne. Enfin, il apparaît que les nouveaux fournisseurs ne connaissent pas la même permanence de salariés-prestataires qu'antérieurement, ce qui suppose un investissement répété des établissements clients dans la relation pour transférer ces compétences.

"Les entreprises plus lointaines viennent moins souvent. Ils nous connaissent moins bien et cela se ressent dès qu'il y a un pépin » [Directeur de l'établissement client T].

"Avec les nouveaux fournisseurs, on voit souvent des nouvelles têtes, et il faut tout ré-expliquer » [Chef de service de l'établissement client P].

En définitive, ce changement a introduit, selon nos interlocuteurs, de nouveaux risques en termes de continuité de l'exploitation avec un système productif décrit comme étant régi par l'urgence et par des dysfonctionnements permanents là où, antérieurement, le système était maîtrisé. On peut ainsi estimer que si la transformation du processus d'achat a contribué à rationaliser les coûts directs d'achats de prestations, elle s'est aussi traduite par des coûts induits et par une perte de «slack organisationnel», qui n'ont pas pour autant donné lieu à une évaluation chiffrée par les acteurs eux-mêmes.

Les implications de la rupture des relations de confiance, très négatives pour les fournisseurs et mitigées pour les clients, nous ont conduit à analyser les fragilités d'un mode de contrôle exclusivement fondé sur l'informel et inscrit dans un réseau local.

\subsection{Une relation de confiance non instrumentée ne passe pas l'épreuve de la réactivation du marché}

\subsubsection{Déficit d'instrumentation du contrôle par la confiance}

Dans le cas étudié, le déplacement du niveau de la prise de décision en matière d'achats de prestations d'entretien et de maintenance s'est simultanément traduit par un changement des critères de la prise de décision. Alors que les acheteurs locaux avaient privilégié la flexibilité opérationnelle et la détention de compétences spécifiques des fournisseurs en cohérence avec la nature de la prestation attendue, les acheteurs centraux ont appliqué des critères de sélection et d'évaluation principalement axés sur les prix, ce qui a réactivé la contrainte marchande et a contribué à modifier les objectifs de la coopération.

En l'absence d'indicateurs chiffrés permettant de démontrer l'apport qualitatif des fournisseurs locaux qui auraient pu justifier un surcoût de leur prestation, le prix était le seul 
critère dont disposaient les acheteurs centraux pour comparer « de loin » les fournisseurs entre eux. Parallèlement, les effets induits du changement de politique d'achats pour les établissements locaux (perte de flexibilité, perte de maîtrise des compétences spécifiques, perte d'un «matelas » organisationnel) n'ont pas été pris en compte dans le processus de rationalisation-centralisation des achats. Ils apparaissent comme des « coûts-performances cachés »-selon le terme employé par Savall et Zardet (1992, p. 39)- qui, n'étant pas mis en évidence par le système d'information, se sont trouvés de ce fait relégués au second rang. Leur émergence introduit néanmoins des doutes quant à la performance globale des choix opérés.

"Au total, on refait machine arrière. Le changement de politique d'achats nous a permis d'améliorer notre trésorerie, mais on ne fait plus correctement notre travail, et ça, on ne sait pas le montrer dans les tableaux de bord» [Directeur de l'établissement client $\mathrm{O}$.

«Maintenant, c'est nous qui faisons moins bien qu'avant, mais on ne sait pas dire combien ça coûte " [Directeur de l'établissement client M].

"On est obsédé par la productivité [le ratio tonnes/hommes] et en même temps, on sait pertinemment que ce n'est plus sur ce ratio que l'on améliorera notre performance. » [Directeur de l'établissement client P]

Ce déficit d'évaluation a posteriori des effets de la substitution d'un mode de contrôle par le marché à un mode de contrôle par la confiance dans les relations client-fournisseur fait écho à l'absence antérieure d'instrumentation de la performance du mode de contrôle par la confiance. Ainsi, une relation locale client-fournisseur fondée sur la confiance qui s'exonère de critères d'évaluation numériques ne permet pas de fournir la preuve de sa performance, ce qui est néanmoins nécessaire dès que le niveau et les critères de décision changent. C'est alors tout le système informel sur lequel repose le contrôle par la confiance qui est remis en question. Ces éléments d'analyse vont dans le sens des travaux de Guibert et Dupuy (1997) qui suggèrent que le contrôle formel et le contrôle informel doivent se développer en cohérence l'un par rapport à l'autre pour garantir la cohésion du système. Dans le cas étudié, l'absence de dispositifs formels a effectivement conduit à fragiliser les relations.

\subsubsection{Changement de réseau de relations et absence de transfert de la relation de confiance}

Les relations entre les établissements locaux et les fournisseurs ont été remises en question avec l'arrivée d'un troisième protagoniste : les directions des achats des groupes industriels. Dans la relation à trois, les établissements ont perdu de leur autonomie de décision en matière d'achat et plusieurs éléments attestent d'une «reprise en mains» des établissements 
industriels par les directions nationales. De même, dans ce changement de réseau social des décisions d'achats, la confiance pré-établie entre établissements clients et fournisseurs locaux n'a plus trouvé sa place qu'à la marge.

Au sein des établissements locaux, cette situation donne lieu à des mécontentements internes, des tentatives de contournement des directives nationales et la multiplication des conflits ouverts avec les acheteurs centraux.

"Maintenant chez eux, le service central des achats et le service technique se font la guerre : les premiers ne pensent qu'au prix mais ne connaissent pas les métiers et les seconds ne sont plus satisfaits des prestataires qu'ils récupèrent » [Directeur de l'entreprise prestataire E].

"Chez nous, les gens râlent. Ils disent qu'ils ne savent pas travailler avec les nouveaux fournisseurs » [Directeur de l'établissement client P].

"On essaye d'arranger encore un peu les choses en confiant des bouts de contrats à nos anciens fournisseurs, mais il ne faut pas dépasser le montant de la délégation de signature » [Directeur de l'établissement client O].

Ainsi, ce cas illustre la fragilité d'un contrôle par la confiance fondé sur les relations interpersonnelles lorsque celle-ci ne se transmet pas au niveau inter-organisationnel. Loin de se généraliser, la confiance locale a conduit à des formes de défiance réciproque au sein des entreprises clientes (entre les directions centrales et les établissements locaux). Plus largement, on peut s'interroger sur la possibilité de développer une confiance interorganisationnelle (définie comme une orientation collective vis-à-vis des individus de l'entreprise partenaire) dans les grandes organisations compte tenu de la multiplicité des acteurs concernés par les relations client-fournisseur, de la diversité de leurs intérêts et des réseaux dans lesquels ils sont inscrits.

\section{Conclusion}

L'évolution des relations client-fournisseur dans la vallée de la Maurienne dans les années quatre-vingt dix est riche d'enseignements sur les processus de rupture de contrôle par la confiance et sur leurs implications directes et indirectes pour les clients et pour les fournisseurs.

Cette étude souligne et approfondit la connaissance des fragilités du contrôle par la confiance. Non instrumenté par des dispositifs formels, ce mode de contrôle ne passe pas l'épreuve du marché. Ainsi, le contrôle par la confiance est paradoxalement puissant et fragile en même 
temps : puissant parce qu'il permet de sélectionner des fournisseurs sur des critères qualitatifs qui ne sont pas mesurables mais qui sont néanmoins importants; fragile parce qu'étant affranchi des dispositifs formels et reposant sur les relations inter-personnelles, il est difficilement transférable du niveau local au niveau organisationnel global; fragile aussi parce que, si la défiance surgit, introduite par un tiers, les bases même du modèle s'effondrent.

Cette étude montre également qu'une relation client-fournisseur n'est pas une relation entre deux boîtes noires, mais entre des individus qui opèrent à des niveaux différents (en local / en central) et dont les intérêts peuvent être divergents. Dès lors, quand on parle des intérêts du client, des intérêts de quel acteur au sein de l'entreprise cliente parle-t-on ? Des acheteurs centraux qui signent les contrats ? Ou des unités locales qui utilisent les prestations des fournisseurs? On voit bien qu'une étude plus fine sur l'organisation du client, sur les différentes parties prenantes et sur leur poids dans les décisions d'achat est nécessaire pour mieux comprendre les relations client-fournisseur. C'est en quelque sorte l'articulation du contrôle intra-organisationnel et du contrôle inter-organisationnel qui est en jeu ici et qui ouvre des perspectives de recherche restant inexplorées à ce jour.

Enfin, elle interroge indirectement les discours des entreprises orientés vers un accroissement de leur responsabilité territoriale et vers l'établissement de relations partenariales pérennes passant par un soutien des fournisseurs, notamment en situation de crise. Dans le cas étudié, les processus observés vont à l'encontre des pratiques de développement durable par ailleurs prônées. Ce constat ouvre alors une autre perspective de recherche : celle de la compréhension des logiques et des formes de développement durable appliqué aux relations inter-entreprises.

\section{Bibliographie}

Adler P.S. (2001), «Market, hierarchy and trust: the knowledge economy and the future of capitalism », Organization Science, vol. 12, pp. 215-234.

Andaleeb S. (1995), «Dependence Relations and the Moderating Role of Trust: Implications for Behavioral Intentions in Marketing Channels », International Journal of Research in Marketing, vol. 12, pp. 157-172.

Aulakh P.S., Kotabe M., Sahay A. (1996), « Trust and Performance in Cross-Border Partnerships : A Behavioral Approach », Journal of International Business Studies, Special Issue, pp. 1105-1132.

Berry M. (1983), Une technologie invisible? L'impact des instruments de gestion sur l'évolution des systèmes humains, Centre de recherche en gestion de l'Ecole Polytechnique.

Brousseau E. (1996), «Contrats et comportements coopératifs : le cas des relations inter-entreprises », in J.-L. Ravix (ed.), Coopération entre les entreprises et organisations industrielles, Paris, CNRS Editions, pp. 23-49. 
Chakravarthy B., Gargiulo M. (1998), «Maintaining leadership legitimacy in the transition to new organizational forms », Journal of Management Studies, vol. 35, n4, July, pp. 437-456.

Chiapello E. (1996), «Les typologies des modes de contrôle et leurs facteurs de contingence : un essai d'organisation de la littérature », Comptabilité Contrôle Audit, tome 2, vol.2, pp. 51-74.

Crozier M., Friedberg E. (1977), L’Acteur et le Système, Paris, Ed. du Seuil.

Das T.K., Teng B.-S. (1998), «Between Trust and Control : Developing Confidence in Partner Cooperation in Alliances », Academy of Management Review, vol. 23, n³, pp. 491-512.

Dekker H.C. (2004), «Control of inter-organizational relationships : evidence on appropriation concerns and coordination requirements », Accounting, Organization and Society, vol. 29, pp. 27-49.

Dumoulin R. (1997), «Marché, bureaucratie et clans dans les réseaux stratégiques », Comptabilité Contrôle Audit, tome 3, vol. 2, pp. 45-58.

Dumoulin R., Gbaka A. (1997), «Contrôle d'entreprises et réseaux stratégiques, une étude exploratoire », Comptabilité Contrôle Audit, tome 3, vol. 1, pp. 23-38.

Dumoulin R., Meschi P.-X. et Uhlig T. (2000), «Management, contrôle et performance des réseaux d'entreprises : étude empirique de 55 réseaux d'alliances », Finance Contrôle Stratégie, vol. 3, n², juin, pp. 81112 .

Dwyer F., Schurr P., Oh S. (1987), « Developing Buyer-Seller Relationships », Journal of Marketing, vol. 51, pp. 11-27.

Dyer J.H., Chu W. (2000), «The Determinants of Trust in Supplier-automaker Relationships in the US, Japan and Korea », Journal of International Business Studies, vol. 31, n², pp. 259-285.

Froehlicher T. (2000), «La dynamique de l'organisation relationnelle : conventions et réseaux sociaux au regard de l'enchevêtrement des modes de coordination », Finance Contrôle Stratégie, vol. 3, n², juin, pp. 113-143.

Ganesan S. (1994), «Determinants of Long-Term Operation in Buyer-Seller Relationships », Journal of Marketing, vol. 58, April, pp. 1-19.

Gorgeu A., Mathieu R. (1983), «Dix ans de relations de sous-traitance dans l'industrie française », Travail, n²8, printemps-été, pp. 23-43.

Gosse B., Sargis-Roussel C. et Sprimont A. (2002), «Les changements organisationnels liés aux stratégies d'externalisation : le cas d'une entreprise industrielle », Finance Contrôle Stratégie, vol. 5, n 1, pp. 101-128.

Granovetter M. (1985), «Economic Action And Social Structure : the Concept of Embeddedness », American Journal of Sociology, vol.91, n`3, pp. 481-510.

Guibert N. et Dupuy Y. (1997), «La complémentarité entre contrôle «formel » et «contrôle informel »: le cas de la relation client-fournisseur », Comptabilité Contrôle Audit, tome 3, vol. 1, pp. 39-52.

Guibert N. (1996), La relation client-fournisseur et les nouvelles technologies de l'information: le rôle des concepts de confiance et d'engagement, thèse de doctorat en sciences de gestion, Université de Montpellier II.

Guibert N. (1999), «La confiance en marketing : fondements et applications », Recherche et Applications en Marketing, vol. 14, $\mathrm{n}^{\circ} 1$, pp. 1-19.

Gulati R. (1995), «Does Familiarity Breed Trust? The Implications of Repeated Ties for Contractual Choice in Alliances », Academy of Management Journal, vol. 38, February, pp. 85-112.

Hakansson H., Lind J. (2004), «Accounting and network coordination », Accounting, Organizations and Society, vol. 29, pp. 51-72.

Heide J.B. (1994), «Interorganizational Governance in Marketing Channels », Journal of Marketing, vol. 58, pp. 71-85.

Karpik L. (1996), « Dispositifs de confiance et engagements crédibles », Sociologie du travail, n4, pp. 527-550.

Langfield-Smith K., Smith D. (2003), «Management control systems and trust in outsourcing relationships », Management Accounting Research, vol. 14, pp. 281-307.

Larson A. (1992), «Network Dyads in Entrepreneurial Settings: A Study of Governance of Exchange Relationships », Administrative Science Quarterly, vol. 37, pp. 76-104. 
Macaulay S. (1963), «Non-Contractual Relations in Business : a Preliminary Study », American Sociological Review, vol. 28, February, pp. 55-67.

Macneil I.R. (1980), The New Social Contract, An Inquiry into Modern Contractual Relations, CT: Yale University Press.

Miles M., Huberman A. (1991), Analyse de données qualitatives, recueil de nouvelles méthodes, Bruxelles, De Boeck Université (1ère édition américaine 1984).

Neuville J.-P. (1997), «La stratégie et la confiance. Le partenariat industriel observé depuis le fournisseur », Sociologie du travail, $\mathrm{n}^{\circ} 3$, pp. 297-319.

Noordewier T.G., John G., Nevin J.R. (1990), «Performance Outcomes of Purchasing Arrangements in Industrial Buyer-Vendor Relationships », Journal of Marketing, vol. 54, October, pp. 80-93.

Nooteboom B., Berger H., Noorderhaven N.G. (1997), «Effects of Trust and Governance on Relational Risk », Academy of Management Journal, vol. 40, n², pp. 308-338.

Ouchi W.G. (1980), «Markets, Bureaucracies and Clans », Administrative Science Quarterly, vol. 25, $\mathrm{n}^{\circ} 1, \mathrm{pp}$ 129-141.

Poppo L., Zenger T. (2002), «Do formal contracts and relational governance function as substitutes or complements ?», Strategic Management Journal, vol. 23, pp. 707-725.

Raveyre M. et Saglio J. (1984), « Les systèmes industriels localisés : éléments pour une analyse sociologique des ensembles de PME industriels », Sociologie du travail, n²-84, pp. 157-176.

Richardson G.B. (1972), « The Organization of Industry », Economic Journal, vol. 82, pp. 883-896.

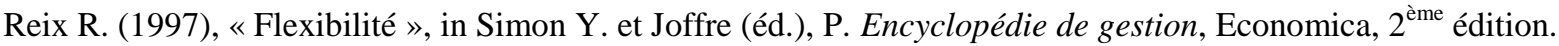

Ring P.S., Van de Ven A.H. (1992), « Structuring Cooperative Relationships between Organizations », Strategic Management Journal, vol. 13, pp. 483-498.

Ring P.S., Van de Ven A.H. (1994), « Development Processs of Cooperative Interorganizational Relationships », Academy of Management Review, vol. 19, n¹, pp. 90-118.

Sako M. (1992), Price, Quality and Trust. Interfirm Relations in Britain and Japan, Cambridge University Press.

Savall H. et Zardet V. (1992), Le nouveau contrôle de gestion, méthode des coûts-performances cachés, Editions comptables Malesherbes, Eyrolles.

Saxton T. (1997), « The effects of partner and relationship characteristics on alliance outcomes », Academy of Management Journal, vol. 40, pp. 443-461.

Van der Meer-Kooistra J., Vosselman G.J. (2000), «Management control of interfirm transactional relationships : the case of industrial renovation and maintenance », Accounting, Organizations and Society, vol. 25, pp. 51-77.

Wathne K.H., Biong H. et Heide J.B. (2001), Choice of Supplier in Embedded Markets : Relationship and Marketing Program Effect, Journal of Marketing, vol. 65, pp. 54-66.

Zaheer A. McEvily B., Perrone V. (1998), «Does trust matter: Exploring the effects of interorganizational and interpersonal trust on performance », Organization Science, vol. 9, n², pp. 141-159.

Zucker G.L. (1986), « Production of Trust : Institutional Sources of Economic Structure, 1840-1920 », in B.M. Staw et L.L. Cumings (ed.), Research in Organization Behavior, vol. 8, Greenwich, CT, JAI Press, pp. 53-111.

Yin R.K. (1989), Case study research : design and methods, Newbury Park, Sage Publications, 2nde édition.

\footnotetext{
${ }^{1}$ Guibert (1999) propose une revue de littérature sur les fondements et applications de la confiance en marketing qui constitue le champ le plus vaste traitant des relations client-fournisseur.

${ }^{2}$ Ouchi (1980) propose une réflexion fondamentale sur le concept de contrôle. Sa typologie est, d'après l'auteur, transposable aux relations inter-organisationnelles. En outre, les relations client-fournisseur, étant initialement dominées par l'informel, elles ne comportent pas «l'héritage bureaucratique » des relations intraorganisationnelles (Guibert et Dupuy, 1997). Ainsi, elles nous semblent particulièrement adaptées à l'étude des différents modes de contrôle, et en particulier à l'étude du contrôle par la confiance.
} 


\footnotetext{
${ }^{3}$ Nous avons construit ce tableau principalement à partir des travaux de Dekker (2004), Dumoulin (1997), Langfield-Smith et Smith (2003) et Van der Meer-Kooistra et Vosselman (2000). Les autres ne donnent pas d'information sur les facteurs de contingence des modes de contrôle.

${ }^{4}$ Le contrôle hiérarchique et le contrôle bureaucratique sont souvent pris l'un pour l'autre. Pour preuve, Hakansson et Lind (2004), qui s'appuient sur la typologie d'Ouchi (1980), parlent de coordination par la hiérarchie, alors qu'Ouchi emploie le terme de bureaucratie.

${ }^{5}$ Das et Teng (1998) emploient le terme « confidence » qui exprime une assurance générale vis-à-vis d'une situation (être confiant).

${ }^{6}$ Cette étude de cas a été réalisée dans le cadre d'une étude pour le ministère de l'Industrie, portant sur «L'analyse des répercussions des nouvelles pratiques d'achats des donneurs d'ordres sur les fournisseurs, dans un bassin d'emploi ».

${ }^{7}$ Les établissements industriels clients de la vallée sont tous des sites de production de groupes dont les sièges sociaux se trouvent en région parisienne.

${ }^{8}$ Il s'agit d'entreprises de moins de 50 salariés. 7 d'entre elles ont été créées dans les années 1960-1970, les autres ont été créées soit antérieurement ( 2 d'entre elles en 1950) soit postérieurement ( 2 d'entre elles dans les années 1980).
} 